\title{
BMJ Open Comparing rates and characteristics of ambulance attendances related to extramedical use of pharmaceutical opioids in Australia: a protocol for a retrospective observational study
}

\author{
Suzanne Nielsen, ${ }^{\oplus 1,2,3}$ Rose Crossin, ${ }^{1,3}$ Melissa Middleton, ${ }^{1}$ Catherine Martin, ${ }^{4}$ \\ James Wilson, ${ }^{3}$ Tina Lam, ${ }^{1}$ Debbie Scott, ${ }^{1,3}$ Karen Smith, ${ }^{1,5,6}$ Dan Lubman ${ }^{1,3}$
}

To cite: Nielsen S, Crossin R, Middleton M, et al. Comparing rates and characteristics of ambulance attendances related to extramedical use of pharmaceutical opioids in Australia: a protocol for a retrospective observational study. BMJ Open 2019;9:e029170. doi:10.1136/ bmjopen-2019-029170

- Prepublication history and additional material for this paper are available online. To view these files, please visit the journal online (http://dx.doi org/10.1136/bmjopen-2019029170).

Received 15 January 2019 Revised 11 April 2019 Accepted 17 April 2019
Check for updates

(C) Author(s) (or their employer(s)) 2019. Re-use permitted under CC BY-NC. No commercial re-use. See rights and permissions. Published by BMJ.

For numbered affiliations see end of article.

Correspondence to A/Prof. Suzanne Nielsen; suzanne.nielsen@monash.edu

\section{ABSTRACT}

Introduction and aims Extramedical use of, and associated harms with pharmaceutical opioids are common. Analysis of coded ambulance clinical records provides a unique opportunity to examine a national population-level indicator of relative harms. This protocol describes an observational study with three aims: (1) to compare supply adjusted rates of pharmaceutical opioid-related ambulance attendances for buprenorphine, codeine, fentanyl, oxycodone, oxycodone-naloxone, morphine, pethidine, tramadol and tapentadol; (2) to compare presentation characteristics for these commonly used pharmaceutical opioids and (3) to describe the context surrounding ambulance presentations related to oxycodone, a widely used opioid with an established abuse liability, and tapentadol, a more recent 'atypical' opioid on the Australian market, with fewer studies that have directly examined signals of extramedical use.

Method Trained coders extract data from clinical records for ambulance presentations relating to extramedical use of commonly used pharmaceutical opioids. These data form the basis of a large, national database that captures alcohol-related and drug-related harms. Supply adjusted rates of presentations will be examined using Poisson regression. Multinomial logistic regression will be used to compare severity and other characteristics of attendances relating to different pharmaceutical opioids. Tapentadol-related and oxycodone-related cases will be qualitatively examined to understand the situationally specific contexts of the ambulance attendances outside of the characteristics captured in routinely coded variables.

Ethics and dissemination Ethics approval related to analysis of ambulance attendance data was obtained from the Eastern Health Human Research Ethics Committee (E122 08-09), with an amendment specific to the qualitative analysis. Findings will be submitted for peer review in 2019. The understanding of risk profiles in realworld settings is of international public health importance. The analysis and publication of findings from this national dataset of clinical records will provide one of the most nuanced analyses to date of relative harms across nine pharmaceutical opioids over a 6 -year period.
Strengths and limitations of this study

- Strengths of this study include the use of coded pharmaceutical opioid-related ambulance attendance data as validated population-level indicator of opioid-related harm (such as extramedical use and overdose) to inform risk profiles in real-world settings.

- We will compare the supply adjusted rates and characteristics of ambulance attendances with commonly used pharmaceutical opioids.

- Limitations include the use of administrative data, and a lack of toxicological data to confirm substances taken.

\section{INTRODUCTION}

Recently, the world's attention has focused on the rapidly escalating opioid-related deaths occurring in North America and other high-income countries. ${ }^{1}$ This has put into sharp focus the need to understand the risk profiles associated with different pharmaceutical opioids.

The number and potency of available pharmaceutical opioids has increased rapidly over the past decades. Morphine, a selective mu-opioid agonist, was isolated $>200$ years ago. ${ }^{2}$ Following this, analogues such as diamorphine and codeine were developed. Later, semi-synthetic opioids such as oxycodone (a full agonist at the mu-opioid receptor) and buprenorphine (a partial agonist at the mu-opioid receptor with activity at delta and kappa) ${ }^{3}$ were isolated. In addition, newer 'atypical' opioids such as tramadol and tapentadol exert their analgesic effects via opioid and non-opioid mechanisms. ${ }^{4}$ Tramadol, a lower potency mu-opioid receptor agonist with a more potent metabolite, also selectively inhibits norepinephrine and serotonin 
uptake. ${ }^{5}$ Tapentadol is another synthetic opioid, structurally similar to tramadol, with mu-opioid receptor agonism and inhibition of norepinephrine reuptake. ${ }^{7}$

A US study of severe adverse events (SAEs) found a positive linear relationship between opioid potency and SAE rate, with the highest rate observed with hydromorphone $(8.02$ SAEs $/ 100 \mathrm{~kg})$, and the lowest rate with tapentadol $(0.27 \mathrm{SAE} / 100 \mathrm{~kg}){ }^{8}{ }^{8}$ This suggests that harms related to opioids may not be equal, although replication studies outside the USA are needed.

Abuse liability studies also find differences between prescribed opioids. Differences in the strength of reinforcing or subjective effects between tramadol, oxycodone, codeine have been found. ${ }^{9}$ Subjective effects of oxycodone appeared greater than codeine, although all opioids examined were reinforcing, particularly at higher doses. ${ }^{9}$ A separate study examined ratings of "I feel high", and the amount that people were willing to pay for a drug also varied by opioid type. ${ }^{10}$ Defined doses of diamorphine, morphine and oxycodone had higher ratings than buprenorphine and fentanyl. ${ }^{10}$ In this study, oxycodone produced robust reinforcing effects, consistent with systematic review of nine studies that oxycodone had a higher abuse liability relative to other opioids. ${ }^{11}$

Although abuse liability may vary in controlled laboratory studies, opioid use in real-world settings can vary as a function of cost, availability and other contextual factors. ${ }^{12}$ For this reason, pharmacovigilance studies are important to monitor for signals of extramedical use in real-world settings. Furthermore, signals of extramedical use may only appear after trials, as those likely to use opioid extramedically are often excluded from these studies, so data from diverse populations are important. ${ }^{13}$

Sentinel surveillance aims to assess non-medical use and harms with opioids such as oxycodone and morphine. ${ }^{14}$ For newer opioids, or opioids infrequently used by sentinel study populations, population-level studies can more completely assess for signals of extramedical use. ${ }^{15}$ One population-level indicator of opioid-related harm is ambulance attendances. These data are recognised as a valuable data source for identifying population-level signals of harm. ${ }^{16}$ In Australia, the clinical records of ambulance attendances related to extramedical use of pharmaceuticals (ie, use outside a medical context, or in a higher dose than prescribed) are coded by trained research assistants. These data can inform the risk profile with different pharmaceutical opioids, ${ }^{17}$ and has been used to monitor harms related to quetiapine and pregabalin. ${ }^{18} 19$ These data can determine if unintended harms such as extramedical use and overdose are emerging, and provide information on the frequency, severity and context of presentations.

As such, this paper outlines the design of a study that aims to conduct a detailed examination of ambulance attendances related to pharmaceutical opioids, and test the hypothesis that the context and frequency of harms with different opioids will vary by opioid type. We write this protocol to maximise transparency. ${ }^{20}{ }^{21}$ The study is supported by an untied educational grant by Seqirus, who make Palexia (tapentadol) and Tramal (tramadol). As has been highlighted by others in the field, ${ }^{22}$ prospectively publishing study protocols with primary aims and related analysis plans assist in establishing independence around the study design, providing transparency and ensuring a commitment to publishing study findings regardless of the outcome. ${ }^{21}$

\section{METHODS}

\section{Study aims}

This study has three aims:

1. Compare the supply adjusted rates of ambulance attendances across commonly used pharmaceutical opioids (buprenorphine, codeine, fentanyl, oxycodone, oxycodone-naloxone, morphine, pethidine, tramadol and tapentadol).

2. Compare presentation characteristics for these nine opioids.

3. Describe the context surrounding ambulance presentations related to two opioids: oxycodone, a widely used opioid with an established abuse liability, and tapentadol, a newer opioid with fewer studies describing extramedical use.

We will answer the following research questions:

1. Do the supply adjusted rates of ambulance presentations differ by opioid potency?

2. Does the severity of presentation (as measured by presenting Glasgow Coma Scale (GCS)) or other characteristics vary by opioid type?

3. Are there differences in the context surrounding ambulance presentations related to extramedical use of oxycodone and tapentadol?

\section{Study design and setting}

Data come from ambulance attendances in Victoria, a state which comprises approximately $26 \%$ of Australia's population, ${ }^{23}$ supplemented by national ambulance data. The Victorian dataset provides complete coverage across the study period (January 2013 until September 2018, excluding 3 months of missing data October-December 2014, due to industrial action). Coded electronic patient care records (ePCR) provide information on acute harms arising from extramedical pharmaceutical use, thus allowing comparison of attendance characteristics across multiple opioids.

We will examine data from the National Ambo Project from Queensland, New South Wales, Australian Capital Territory, Northern Territory and Tasmania to determine if Victorian trends are comparable to national trends. National data are available in quarterly 'snap-shot months' (table 1) and are screened and coded using the same procedures and coders as the Victorian data. See figure 1 for an overview of data sources and study processes. We will aim to complete case identification by March 2019, with analyses planned for March-April 2019. 


\begin{tabular}{ll}
\hline $\begin{array}{l}\text { Table 1 } \\
\text { State/ } \\
\text { Territory }\end{array}$ & Summary of data availability across jurisdictions \\
\hline ACT & $\begin{array}{l}\text { Notes on data available* } \\
\text { March } 2015 \text { until December } 2017\end{array}$ \\
NSW & $\begin{array}{l}\text { All cases coded 1 month per quarter from } \\
\text { March } 2015 \text { until December 2017 }\end{array}$ \\
NT & $\begin{array}{l}\text { All cases coded 1 month per quarter from } \\
\text { March 2016 until December 2017 }\end{array}$ \\
QLD & $\begin{array}{l}\text { All cases coded 1 month per quarter from } \\
\text { March 2015 until December 2016 }\end{array}$ \\
TAS & $\begin{array}{l}\text { All cases coded 1 month per quarter from } \\
\text { March 2014, until December 2017 }\end{array}$ \\
VIC & $\begin{array}{l}\text { All cases coded from January 2012 till } \\
\text { September 2018 }\end{array}$ \\
\hline WA and SA & Data not yet available \\
\hline
\end{tabular}

*We will conduct a comparison on supply adjusted rates of attendance for VIC and other states using periods of time where corresponding data are available.

ACT, Australian Capital Territory; NSW, New South Wales; NT, Northern Territory; QLD, Queensland; SA, South Australia; TAS, Tasmania; VIC, Victoria; WA, Western Australia.

\section{Pharmaceutical opioid sales data}

We will estimate the amount of each opioid supplied using monthly sales data (IQVIA third-party access programme). The total amount of each opioid will be calculated by jurisdiction in $\mathrm{mg}$, converted into oral morphine equivalents (OME), ${ }^{24}$ and used to calculate a supply adjusted rate of attendances, consistent with previous studies of pharmaceutical opioid-related harm that have adjusted for supply using similar methods. ${ }^{825}$

\section{Ambulance attendance data}

Ambulance data are collected as part of the Ambo Project, a collaboration between Turning Point, Monash University and Ambulance Victoria (AV). Methods for the Ambo Project have been previously described, ${ }^{18}{ }^{19}$ and are outlined below.

Data include ePCR, computer-aided dispatch notes and clinical details associated with the attendance that provide part of the patient care record (eg, records of respiration rate and GCS). Primary filtering based on over-inclusive keyword searches identifies attendances with involvement of alcohol, other drugs or mental health symptomology. This filtered dataset is provided to Turning Point where data cleaning, validation and coding of the ePCR is performed by a specialist team of research assistants. Details on research assistant training, and inter-rater review processes are contained in online supplementary appendix A. A systematic and validated coding system captures information from the ePCR. The core criterion used in determining the involvement of a substance is: "Is it reasonable to attribute the immediate or recent (not merely chronic) overappropriate or inappropriate (ie, extramedical) ingestion of the substance or medication as significantly contributing to the reason for the ambulance attendance?", as determined through examination of the clinical notes. As such, this dataset captures information on acute, not chronic, harms.

For this study, ambulance attendances where extramedical pharmaceutical opioid use was identified as a significant contributor to an attendance will be included. This dataset excludes cases of solely therapeutic use. Pharmaceutical opioid-related attendances include those where; a person who is prescribed opioids consumed medication more often or in higher than recommended amounts, a person consumed opioids not prescribed to them, or if opioids were consumed with a combination of other substances that contributed to the ambulance attendance. The inclusion criteria mean that extramedical opioid use must significantly contribute to the attendance, however other drugs or substances may have also been overused or inappropriately consumed and the extramedical opioid use may not be the primary or only reason for the ambulance attending. These criteria differentiate Ambo Project data from adverse event reporting from the Therapeutic Goods Administration Database of Adverse Event (AE) Notifications, ${ }^{26}$ which captures spontaneous AEs from prescribed medications. Spontaneous $\mathrm{AE}$ reporting can effectively identify serious harms with prescribed medicines, although is recognised to miss the vast majority of AEs. ${ }^{27}$

Other associated factors such as alcohol and other drug use, mental health symptoms and self-harm are also coded. All illicit drug use is coded regardless of quantity. Current mental health symptomology is coded rather than mental health diagnosis as paramedics do not screen or assess mental illness diagnoses during an ambulance attendance.

These methods are consistent across other jurisdictions, which collect quarterly data as part of the National Ambo Project, with the exception that ambulance services in Tasmania, Australian Capital Territory and the Northern Territory provide all data and do not undertake primary filtering.

All substances related to the attendance are coded; including alcohol, illicit and pharmaceutical drugs. For this project, we will examine buprenorphine (as a single ingredient), codeine (codeine as a single ingredient, and in combination with paracetamol, or ibuprofen, or aspirin), fentanyl, morphine, oxycodone, oxycodone-naloxone, pethidine, tapentadol and tramadol. We will exclude opioids used as treatments for opioid dependence (methadone, buprenorphine-naloxone or buprenorphine and a single ingredient where indicated that it is for treatment of opioid dependence) as these represent a different clinical indication and specific treatment population.

Buprenorphine, codeine, fentanyl, oxycodone, oxycodone-naloxone, morphine, pethidine and tramadol are routinely coded in the Ambo Project database. Cases involving tapentadol are routinely coded in an 'other opioid' category. For this study, 'tapentadol-related' cases 


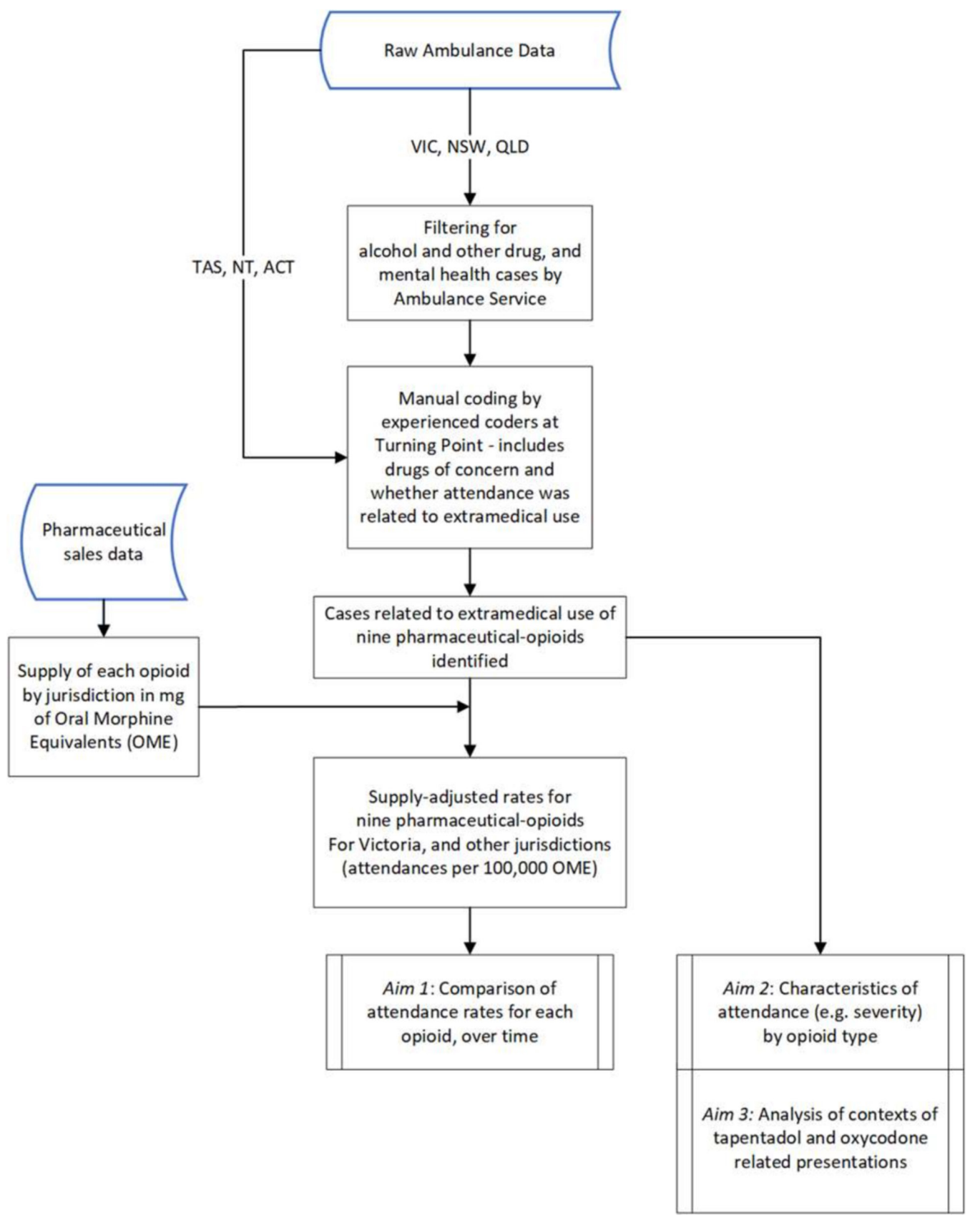

Figure 1 Overview of study processes and data sources. ACT, Australian Capital Territory; NSW, New South Wales; NT, Northern Territory; QLD, Queensland; TAS, Tasmania; VIC, Victoria.

will be identified and coded by searching all 'other opioid' cases from June 2013 (aligned with tapentadol availability as a subsidised medicine in Australia), until September 2018. This is identical to the coding process used with other opioids, the only difference being that the cases will be coded retrospectively from 'other opioids' to 'tapentadol' where there is evidence of tapentadol involvement in the ePCR.

\section{Aim 1: supply adjusted trends 2013-2018}

Analysis of supply adjusted trends will occur by quarterly aggregation of attendances in Victoria, by opioid group, with further aggregation to half-yearly if required to preserve cell sizes of at least five in the majority of cells.
Where cell sizes of $<5$ occur, to preserve anonymity we will report average of all cells with 1-4 cases, rather than the number of cases in that cell. Units will be attendances per $100000 \mathrm{mg} \mathrm{OME}{ }^{24}$

\section{Aim 1 analysis plan}

Prescription opioid-related ambulance attendances will be aggregated into 3 monthly periods corresponding to yearly quarters. Basic descriptive statistical analyses will be used to explore these attendances in Victoria, analyses include frequencies (number of attendances), proportions (demographics) and supply adjusted rates.

Poisson regression will be used to assess trends in supply adjusted rates for Victoria. Regression models will 
be fitted (one for each opioid), and adjusted for quarterly and yearly trends with the number of days per month used to offset the regression. Rates will be calculated for Victoria (where completed data are available), and compared with rates observed in other states for the time periods that data are available (table 1). Due to industrial action, September 2014 contains partially complete data (26 out of 30 days). Supply data for this month will be weighted to represent the average supply for the proportion of days provided for September, with the offset variable representing the total number of days where data have been supplied.

The intention is to assess trends within each opioid where the opioid has been solely indicated, as well as a 'multiple opioid' category representing cases where multiple opioids have been indicated. However, where this is not possible due to small case numbers, trends will be assessed using all cases in which the opioid has been indicated (sole use and multiple opioids).

\section{Aim 2: characteristics of attendances}

For this analysis, opioid-related attendances will be analysed by opioid type listed above, by characteristics as per table 2 (additional details on variables are contained in online supplementary appendix B).

\section{Aim 2 analysis plan}

Multinomial logistic regression will be used to analyse characteristics of opioid-related attendances by opioid type. Opioid type (sole use of each opioid and multiple opioid use) will be used as the dependent variable, with each category being compared with morphine as mid-potency opioid, regressed on each characteristic separately. If the number of morphine cases is insufficient to allow use as a reference category then oxycodone will become the reference category. Each model will be adjusted for using a collapsed state variable (where jurisdictions with expected fewer cases such as the Australian Capital Territory and Northern Territory will be aggregated into an 'other states' category). When considering GCS as a proxy for severity, the model will be further adjusted for age, gender, concurrent alcohol use, concurrent illicit drug use (excluding heroin), concurrent heroin use and concurrent non-opioid pharmaceutical use. All other models will be further adjusted for age, gender and other substance use (as an aggregated variable of concurrent alcohol use, illicit drug use, heroin and non-opioid pharmaceutical misuse). Results will be reported as ORs. Comparison between states will be analysed through an interaction between state and characteristic.

\section{Aim 3: qualitative analysis of contexts of oxycodone-related and tapentadol-related attendances}

Detail from the free-text fields from the ePCR will be used to establish a more complete picture of the ambulance attendance. These data are subject to rigorous coding by a highly trained team of coders using well-established and tested coding frameworks for existing fields, however additional uncoded data available in these free-text fields are rarely used for qualitative analyses. Some studies have used text-based information to explore specific phenomena in further detail, such as intentional and unintentional injury ${ }^{28}$ and heroin overdose. ${ }^{29}$

We will use text-based case descriptions of oxycodone-related and tapentadol-related cases. These will be qualitatively examined in order to provide insight into the situationally specific contexts of the ambulance attendances outside of the characteristics captured in routinely coded variables. We will compare tapentadol-related cases (with tapentadol being a newer 'atypical' opioid with a suggested lower rate of associated adverse events ${ }^{8}$ and less non-medical use $^{30}$ ), to oxycodone-related attendances, as oxycodone is the predominant opioid-analgesic prescribed in Australia ${ }^{31}$ and has a well-recognised abuse liability. ${ }^{11}$ We will consider the details of circumstances surrounding the presentation including details provided on the role and effect of the opioid used.

\section{Aim 3 analysis plan}

Qualitative coding will occur in two stages. First, an initial group of randomly selected cases (estimated $n=30-40$ until saturation has occurred) will be examined by two researchers to independently determine a coding framework. The researchers will negotiate a consensus framework, then code the remaining cases using this agreed on framework. The project team will convene regularly to discuss coding techniques as they develop as well as emerging themes. A $10 \%$ sample of cases will be checked by a third researcher to confirm coding consistency, with further cases reviewed if inconsistencies are identified.

We will examine an equal number of tapentadol-related and oxycodone-related cases, based on the number of cases identified for tapentadol (the less frequently used opioid). We will use a random number generator via Excel to randomly select an equal size number of oxycodone-related cases for qualitative comparison (ie, the same number of cases as in the tapentadol-related case group), and perform the same analyses for oxycodone-related presentations. To ensure that selected oxycodone cases represent all oxycodone cases the subgroup of oxycodone-related attendances will be selected by weighted random sampling, with weightings on the basis of sex, age group, jurisdiction and if the attendance was related to self-harm. Cases involving both tapentadol and oxycodone will be considered as a separate group.

In addition to qualitative analysis, multinomial regression will be used to analyse coded variables representing different aspects of attendance context surrounding three mutually exclusive groups: (1) tapentadol-related cases, (2) oxycodone-related cases and (3) cases that are related to the concomitant use of tapentadol and oxycodone (ie, considered-related to both opioids). Results will be reported as ORs.

All quantitative analysis for aims 1-3 will be conducted in STATA V.13 (StataCorp, College Station, Texas, USA), with $p$ values $<0.05$ considered to be statistically significant. 
Table 2 Variables and response options to be examined in association with pharmaceutical opioid-related ambulance attendances

\begin{tabular}{|c|c|}
\hline Variable & Response options \\
\hline Presenting Glasgow Coma Scale & $\begin{array}{l}3 \text { (non-responsive), 4-8 (severe impairment), 9-12 (moderate impairment), 13-15 } \\
\text { (minor-no impairment) }\end{array}$ \\
\hline $\begin{array}{l}\text { Presenting respiratory rate (breaths per } \\
\text { min) }\end{array}$ & $<6,6-12,>12$ \\
\hline Transport to hospital & Not transported, transported \\
\hline Naloxone administered: not stated, yes & Not stated, yes \\
\hline $\begin{array}{l}\text { Naloxone response: not effective, } \\
\text { effective }\end{array}$ & Not effective, effective \\
\hline Sex of patient & Male, female, other/unspecified \\
\hline Age of patient & $12-34,35-54,55-65$ (nearing retirement age) and $65+^{*}$ \\
\hline $\begin{array}{l}\text { Socioeconomic status based on } \\
\text { residential postcode }\end{array}$ & Quintile $1-5$ based on SEIFA-IRSD 201 s, IRSD $2016^{38}$ \\
\hline Concurrent alcohol involvement & Not stated, alcohol involved but no evidence of intoxication, alcohol intoxicationt \\
\hline Concurrent heroin involvement & Not stated, present \\
\hline $\begin{array}{l}\text { Concurrent illicit drug use (excluding } \\
\text { heroin) }\end{array}$ & $\begin{array}{l}\text { Based on the presence of at least one of the illicit drugs coded for } \\
\text { meth(amphetamine), cannabis, synthetic cannabinoids, emerging psychoactive } \\
\text { substances, cocaine, 3,4-methylenedioxy-methamphetamine (MDMA), gamma- } \\
\text { hydroxybutyrate, ketamine, Lysergic acid diethylamide (LSD), psilocybin, inhalant, } \\
\text { illicit drug other or unspecified) }\end{array}$ \\
\hline $\begin{array}{l}\text { Concurrent non-opioid extramedical } \\
\text { pharmaceutical use }\end{array}$ & $\begin{array}{l}\text { Not stated, present (based on the presence of at least one of the pharmaceutical } \\
\text { groups coded for non-opioid analgesics, benzodiazepines, antidepressants, } \\
\text { antipsychotics, anticonvulsants, opioid-dependence treatments, pharmaceutical } \\
\text { stimulants, other medication) }\end{array}$ \\
\hline Comorbid mental health symptoms & $\begin{array}{l}\text { Not stated, present (based on the presence of at least one of symptoms of anxiety, } \\
\text { depression, psychosis, social/emotional distress, symptoms associated with } \\
\text { disorders with clinical evidence and mental health unspecified) }\end{array}$ \\
\hline $\begin{array}{l}\text { Comorbid suicidal thoughts or } \\
\text { behaviours }\end{array}$ & $\begin{array}{l}\text { Not stated, present (based on the presence of at least one of suicidal ideation, } \\
\text { suicide attempt) }\end{array}$ \\
\hline Comorbid non-suicidal self-injury & $\begin{array}{l}\text { Not stated, present (based on the presence of at least one of threat of non-suicidal } \\
\text { self-injury, non-suicidal self-injury) }\end{array}$ \\
\hline Accidental overdose & Not stated, yes \\
\hline Unknown intent overdose $\ddagger$ & Not stated, yes \\
\hline History of psychiatric issues & $\begin{array}{l}\text { Not stated, present (based on the presence of at least one of history of mood } \\
\text { disorder, psychosis, suicidal ideation, suicide attempt, alcohol and other drug } \\
\text { misuse) }\end{array}$ \\
\hline
\end{tabular}

*Based on previous age categories used in studies of opioid use for pain. ${ }^{39}$ We will exclude cases where age is reported to be $<12$ years due to the unclear intention of use in children of this age, consistent with previous research. ${ }^{4041}$

†The involvement of alcohol is coded as 'alcohol involved' and 'alcohol intoxication'. Attendances where the person has consumed alcohol, but the paramedic notes do not clearly indicate alcohol intoxication are coded as 'alcohol involved' and 'alcohol intoxication is a subset of 'alcohol involved'. The default code is for 'alcohol involved' unless the paramedic notes provide clear evidence of alcohol intoxication. $\ddagger$ Where information provided in the patient care records by the paramedic means that the coding team cannot determine if the overdose was accidental or if there was suicidal intent.

Qualitative data will be managed and analysed using NVivo V.12.0, ${ }^{32}$ but any subsequent quantitative analyses conducted using STATA as above.

\section{Handling known or expected missing data}

Where there are missing data for the characteristics of attendances, we will report the available sample size for each variable. To enable calculation of rates for each quarter with potentially identifiable cases (cell size
1-4), we will impute a value representing the mean for all potentially identifiable cases. This will preserve the total number of cases without compromising privacy of individuals.

There are 3 months of missing Victorian data due to industrial action (October-December 2014) with partial missing data (4 days) for September 2014. For these months, to enable calculation of rates (aim 1), 
we will impute values based on data from September to December in the previous and following year (2013 and 2015). In a planned sensitivity analysis, we will compare results using imputed data to results excluding missing data and report any differences observed.

\section{Ethics and dissemination}

Consistent with the ethics approval, cells of $<5$ will not be reported, although zeroes will be preserved. Due to the sensitivity and potentially identifiable nature of the data line item data are not available for sharing, consistent with the ethics approval.

We will present project findings at relevant scientific conferences. We plan to submit findings for publication as two peer-reviewed journal articles. One article will incorporate the quantitative analysis (aims 1 and 2) and one paper report the qualitative analysis (aim 3). We will report findings in accordance with the REporting of studies Conducted using Observational Routinely collected health Data statement, an extension of the Strengthening the Reporting of Observational Studies in Epidemiology statement for reporting items specific to observational studies using routinely collected health data. ${ }^{33}$

\section{Patient and public involvement}

Interpretation of the findings and dissemination will be informed by people with lived experience through contact with consumer organisations such as the Association of Participating Service Users.

\section{DISCUSSION}

\section{Strengths and limitations}

Recommendations for methods for pharmacovigilance studies identified a number of important features, depending on the aims of the study and the methods employed. ${ }^{16}$ These include the prospective publication of a study protocol including a detailed statistical analysis plan, transparency around study funding and publication in open-access journals. This study will conform to these requirements.

This study has several strengths. First, coded ambulance data capture a broader range of outcomes and a wider population of people with extramedical pharmaceutical opioid use than may be captured in abuse liability studies, clinical trials or spontaneous AE reporting systems.

Second, this study's population-level database accurately codes detailed information about each attendance. This level of detail can provide important insights into harms related to substance use. ${ }^{17-19}{ }^{34-36}$ This study will represent an extension of these studies to provide a detailed analysis of rates and characteristics of harms related with widely used pharmaceutical opioids, and is strengthened by the use of population-level supply data to calculate supply adjusted rates. Finally, the study will be first to provide detailed context around circumstances of pharmaceutical opioid-related ambulance attendances, using coding of qualitative data.
There are limitations in the use of data collected for operational purposes, similar to with hospital inpatient and emergency department data. There is potential for biases to exist in collection and coding, and incomplete or inconsistent recording of variables. Substance involvement is determined from patient-provided information, others at the scene, or paramedic's clinical assessment, not from toxicological testing. Other clinical details (eg, mental health symptoms) are also determined in this way.

Rates of ambulance attendances are calculated based on sales data. Due to frequent ordering and limited capacity for controlled drug storage at the community pharmacy level, sales closely approximate supply. Use of sales data addresses limitations with publicly funded prescription data, which omits privately purchased prescriptions and over-the-counter medications and therefore is an incomplete measure of community opioid supply. Finally, as this is a naturalistic study, we cannot know if different patient populations are more likely to receive a given opioid. As such, there may be unmeasured confounders. Randomised trials can address this limitation; however, patients who use opioids extramedically are usually excluded from trials.

\section{Data statement}

To protect privacy and confidentiality, data from the Ambo Project are provided to Turning Point under strict conditions for the storage, retention and use of the data. The current approval permits storage of the data at one site, Turning Point, with any analysis to be undertaken onsite, no data to be removed and no dissemination of unit level data. Researchers wishing to undertake additional analyses of the data are invited to contact Turning Point as the data custodians.

\section{CONCLUSION}

This will be the first detailed study to compare coded ambulance ePCR as a population-level indicator of prescription opioid-related harm. These data will complement ongoing studies examining extramedical use of tapentadol in sentinel populations of people who use drugs, and data from poisons information centres. ${ }^{37}$ The data in this study represent all acute presentations in community settings where extramedical use of a pharmaceutical opioid analgesic is considered related to the ambulance attendance. This unique dataset has national reach and demonstrated consistency and completeness over multiple years. It will provide one of the most complete analyses of relative harms due to extramedical use for a range of pharmaceutical opioids to date. The study will add to our knowledge, and lead to a more nuanced understanding of whether different pharmaceutical opioids are associated with different harms.

\section{Author affiliations}

${ }^{1}$ Monash Addiction Research Centre, Eastern Health Clinical School, Monash University, Melbourne, Victoria, Australia 
${ }^{2}$ National Drug and Alcohol Research Centre, UNSW Sydney, Randwick, New South Wales, Australia

${ }^{3}$ Turning Point, Monash University Eastern Health Clinical School, North Richmond, Victoria, Australia

${ }^{4}$ Biostatistics Unit, Public Health and Preventative Medicine, Monash University, Melbourne, New South Wales, Australia

${ }^{5}$ Centre for Research and Evaluation, Ambulance Victoria, Doncaster, Victoria, Australia

${ }^{6}$ Department of Community Emergency Health and Paramedic Practice, Monash University, Frankston, Victoria, Australia

Acknowledgements The authors would like to thank Sharon Matthews and the population health team of coders who code the data, and the ambulance services around Australia who provide the data.

Contributors SN initially conceptualised the study and drafted the initial design, with input from RC, DS and JW. CM and MM provided input on, and drafted the statistical analysis plan. SN, RC, DS, JW, TL, KS and DL contributed the drafting of the protocol manuscript and revisions of the protocol. KS, DL and DS lead the broader Victorian Ambo Project and the National Ambo project from which the data are drawn and provided detailed technical advice relating to procedures from that study to inform the development of this study and analysis of the data.

Funding The study is funded by an untied educational grant from Seqirus. SN is the recipient of an NHMRC Career Development Fellowship (\#1163961). The Ambo Project andNational Ambo Project are funded by the Victorian Department of Health andHuman Services, and the Commonwealth Department of Health.

Disclaimer The funders will have no role in the study design, study conduct, analysis or data interpretation. Prior to publication, representatives from Seqirus will have the opportunity to review the manuscript and provide comment on factual inaccuracies, if identified.

Competing interests In the past 5 years, SN has been an investigator on untied education grants from Indivior, unrelated to the current work. SN has provided training to healthcare professionals on identifying and treating codeine dependence for which her institution has received payment from Indivior. DL has received speaking honoraria from the following: AstraZeneca, Indivior, Janssen-Cilag, Lundbeck, Servier and Shire, and has participated on Advisory Boards for Indivior and Lundbeck.

\section{Patient consent for publication Not required.}

Ethics approval Ethics approval related to analysis of ambulance attendance data was obtained from the Eastern Health Human Research Ethics Committee (E122 08-09), with an amendment specific to the qualitative analysis.

Provenance and peer review Not commissioned; externally peer reviewed.

Open access This is an open access article distributed in accordance with the Creative Commons Attribution Non Commercial (CC BY-NC 4.0) license, which permits others to distribute, remix, adapt, build upon this work non-commercially, and license their derivative works on different terms, provided the original work is properly cited, appropriate credit is given, any changes made indicated, and the use is non-commercial. See: http://creativecommons.org/licenses/by-nc/4.0/.

\section{REFERENCES}

1. Seth P, Scholl L, Rudd RA, et al. Overdose deaths involving opioids, cocaine, and psychostimulants - United States, 2015-2016. Am J Transplant 2018;18:1556-68.

2. Krishnamurti C, Rao SC. The isolation of morphine by Serturner. Indian J Anaesth 2016;60:861-2.

3. Pasternak GW, Pan YX. Mu opioids and their receptors: evolution of a concept. Pharmacol Rev 2013;65:1257-317.

4. Pergolizzi JV, LeQuang JA, Taylor R, et al. Designing safer analgesics: a focus on $\mu$-opioid receptor pathways. Expert Opin Drug Discov 2018;13:965-72.

5. Miotto K, Cho AK, Khalil MA, et al. Trends in Tramadol: Pharmacology, Metabolism, and Misuse. Anesth Analg 2017:124:44-51.

6. Bravo L, Mico JA, Berrocoso E. Discovery and development of tramadol for the treatment of pain. Expert Opin Drug Discov 2017;12:1281-91.

7. Vadivelu N, Timchenko A, Huang Y, et al. Tapentadol extendedrelease for treatment of chronic pain: a review. J Pain Res 2011;4:211-8.
8. Murphy DL, Lebin JA, Severtson SG, et al. Comparative Rates of Mortality and Serious Adverse Effects Among Commonly Prescribed Opioid Analgesics. Drug Saf 2018;41:787-95.

9. Babalonis S, Lofwall MR, Nuzzo PA, et al. Abuse liability and reinforcing efficacy of oral tramadol in humans. Drug Alcohol Depend 2013;129(1-2):116-24.

10. Comer SD, Sullivan MA, Whittington RA, et al. Abuse liability of prescription opioids compared to heroin in morphine-maintained heroin abusers. Neuropsychopharmacology 2008;33:1179-91.

11. Wightman R, Perrone J, Portelli I, et al. Likeability and abuse liability of commonly prescribed opioids. J Med Toxicol 2012;8:335-40.

12. Cicero TJ, Ellis MS, Surratt HL, et al. The changing face of heroin use in the United States: a retrospective analysis of the past 50 years. JAMA Psychiatry 2014;71:821-6.

13. Turk DC, O'Connor $\mathrm{AB}$, Dworkin $\mathrm{RH}$, et al. Research design considerations for clinical studies of abuse-deterrent opioid analgesics: IMMPACT recommendations. Pain 2012;153:1997-2008.

14. Peacock AG. Australian Drug Trends 2018. Key findings from the National Illicit Drug Reporting System (IDRS) Interviews. Sydney: National Drug and Alcohol Research Centre, UNSW Australia, 2018.

15. Cicero TJ, Dart RC, Inciardi JA, et al. The development of a comprehensive risk-management program for prescription opioid analgesics: researched abuse, diversion and addiction-related surveillance (RADARS). Pain Med 2007;8:157-70.

16. Peacock A, Larance B, Bruno R, et al. Post-marketing studies of pharmaceutical opioid abuse-deterrent formulations: a framework for research design and reporting. Addiction 2019;114:389-99.

17. Nielsen S, Dietze P, Cantwell K, Lee N, et al. Methadone- and buprenorphine-related ambulance attendances: a population-based indicator of adverse events. J Subst Abuse Treat 2008;35:457-61.

18. Heilbronn C, Lloyd B, McElwee P, et al. Trends in quetiapine use and non-fatal quetiapine-related ambulance attendances. Drug Alcohol Rev 2013;32:405-11.

19. Crossin R, Scott D, Arunogiri S, et al. Pregabalin misuse-related ambulance attendances in Victoria, 2012-2017: characteristics of patients and attendances. Med J Aust 2019;210:75-9.

20. -e.PLOS Medicine Editors. Observational studies: getting clear about transparency. PLoS Med 2014;11:e1001711.

21. West R. Trial protocols. Addiction 2012;107:1544.

22. Degenhardt L, Larance B, Bruno R, et al. Evaluating the potential impact of a reformulated version of oxycodone upon tampering, non-adherence and diversion of opioids: the National Opioid Medications Abuse Deterrence (NOMAD) study protocol. Addiction 2015;110:226-37.

23. Australian Bureau of Statistics. 3101.0 - Australian Demographic Statistics, Mar 2018. 2018 http://www.abs.gov.au/ausstats/abs@.nsf/ $\mathrm{mf} / 3101.0$.

24. Nielsen S, Degenhardt L, Hoban B, et al. A synthesis of oral morphine equivalents (OME) for opioid utilisation studies. Pharmacoepidemiol Drug Saf 2016;25:733-7.

25. Roxburgh A, Hall WD, Dobbins T, et al. Trends in heroin and pharmaceutical opioid overdose deaths in Australia. Drug Alcohol Depend 2017;179:291-8.

26. Therapeutic Goods Administration. Database of Adverse Event Notifications - medicines. Canberra, 2018.

27. Murff HJ, Patel VL, Hripcsak G, et al. Detecting adverse events for patient safety research: a review of current methodologies. J Biomed Inform 2003;36:131-43.

28. Backe SN, Andersson R. Monitoring the "tip of the iceberg": ambulance records as a source of injury surveillance. Scand J Public Health 2008;36:250-7.

29. Bammer G, Ostini R, Sengoz A. Using ambulance service records to examine nonfatal heroin overdoses. Aust $J$ Public Health 1995;19:316-7.

30. Butler SF, McNaughton EC, Black RA. Tapentadol abuse potential: a postmarketing evaluation using a sample of individuals evaluated for substance abuse treatment. Pain Med 2015;16:119-30.

31. Degenhardt L, Gisev N, Cama E, et al. The extent and correlates of community-based pharmaceutical opioid utilisation in Australia. Pharmacoepidemiol Drug Saf 2016;25:521-38.

32. Bazeley P, Jackson K. Qualitative Data Analysis with NVivo. 2nd ed. London: Sage, 2013.

33. Benchimol El, Smeeth L, Guttmann A, et al. The REporting of studies Conducted using Observational Routinely-collected health Data (RECORD) Statement. PLoS Med 2015;12:e1001885.

34. Lloyd BK, McElwee PR. Trends over time in characteristics of pharmaceutical drug-related ambulance attendances in Melbourne. Drug Alcohol Rev 2011;30:271-80.

35. Dietze P, Jolley D, Cvetkovski S, et al. Characteristics of non-fatal opioid overdoses attended by ambulance services in Australia. Aust N Z J Public Health 2004;28:569-75. 
36. Dietze PM, Cvetkovski S, Barratt MJ, et al. Patterns and incidence of gamma-hydroxybutyrate (GHB)-related ambulance attendances in Melbourne, Victoria. Med J Aust 2008;188:709-11.

37. Peacock A, Larance B, Farrell M, et al. Opioid use and harms associated with a sustained-release tapentadol formulation: a postmarketing study protocol. BMJ Open 2018;8:e020006.

38. Australian Bureau of Statistics. 2033.0.55.001 - Census of Population and Housing: Socio-Economic Indexes for Areas (SEIFA), Australia, 2016. Canberra, 2018
39. Campbell G, Nielsen S, Bruno R, et al. The Pain and Opioids IN Treatment study: characteristics of a cohort using opioids to manage chronic non-cancer pain. Pain 2015;156:231-42.

40. Rhodes AE, Bethell J, Spence J, et al. Age-sex differences in medicinal self-poisonings: a population-based study of deliberate intent and medical severity. Soc Psychiatry Psychiatr Epidemiol 2008:43:642-52.

41. Gunnell D, Ho D, Murray V. Medical management of deliberate drug overdose: a neglected area for suicide prevention? Emerg Med $J$ 2004;21:35-8. 